IBIMA Publishing

Journal of e-health Management

https://ibimapublishing.com/articles/JEHM/2020/783101/

Vol. 2020 (2020), Article ID 783101, 12 pages, ISSN : 2165-9478

DOI: $10.5171 / 2020.783101$

Research Article

\title{
Managing Patient Engagement in the Process Approach
}

\section{Małgorzata DYMYT and Marta WINCEWICZ-BOSY}

\author{
General Tadeusz Kosciuszko Military University of Land Forces, Wroclaw, Poland \\ Correspondence should be addressed to: Małgorzata DYMYT; malgorzata.dymyt@awl.edu.pl \\ Received date: 25 February 2020; Accepted date: 17 July 2020; published date: 31 August 2020 \\ Academic Editor: Marta Starostka-Patyk
}

Copyright (C) 2020. Małgorzata DYMYT and Marta WINCEWICZ-BOSY. Distributed under Creative Commons Attribution 4.0 International CC-BY 4.0

\begin{abstract}
The main goal of the article is to identify the essence, role, premises and conditions of managing patient engagement in the process approach. Patient engagement is an important component of health services, covering a wide area of action at the systemic and organizational levels. A comprehensive and coherent approach to the processes related to patient involvement, especially in the area of care decision-making, is essential for the smooth functioning of the healthcare system. The article is of a conceptual research nature and was developed as a result of an in-depth analysis of literature, documents and interviews. During the analysis and evaluation of the examined process, mapping methods were used, enabling the development of a graphic form of the model. For this purpose, iGRAFX software was used. Based on the conducted analyses, a conceptual model of managing the patient engagement processes was proposed, taking into account the main tasks and participants responsible for their implementation at the organizational level. Including patient engagement management in a process context allows you to focus on creating and delivering value to patients and the healthcare system. The process approach to managing this issue allows for understanding the role of the patient in the healthcare system and ensuring the integrity of the applied principles and practices necessary to implement the concept of patient engagement at all levels of the healthcare system. Due to the specificity of health services, the involvement of patients should be directed at conscious and active multilevel cooperation for health.
\end{abstract}

Keywords: Patient Engagement, Health Care System, Health Care Management, Process Management.

\section{Introduction}

The smooth functioning of the health care system is a challenge for policy makers responsible for social policy. In the face of globalisation challenges, especially of a social, demographic, environmental, technological, economic, as well as political and legal nature, health care systems are forced to search for new concepts,

Cite this Article as: Małgorzata DYMYT and Marta WINCEWICZ-BOSY (2020)," Managing Patient Engagement in the Process Approach", Journal of e-health Management, Vol. 2020 (2020), Article ID 783101, DOI:10.5171/2020.783101 
approaches and methods of action to ensure effectiveness. According to the World Health Organization, the health care system includes all activities whose main purpose is to promote, restore or maintain health (WHO, 2000). In this context, the system's activity has a wide, complex and multi-faceted scope and requires the participation of many entities at various levels: macro (system level), meso (interorganisational) and micro (institutional directly related to the performance of activities). In a broad sense, "health care system is a whole of political, economic and cultural, technical and organisational factors, relations, processes and elements, in which individuals, groups and communities interrelate, having the goal to satisfy their health needs" (Kovacic and Jaksic, 2013, p. 101). The main components of the effectiveness of the health care system include patient health status, patient satisfaction and access (availability, utilisation, timeliness) and quality of care (efficacy, safety, continuity) (Kruk and Freedman, 2008). In a well-functioning health system, the provision of services should have the following features: comprehensiveness, accessibility, coverage, continuity, quality, person-centeredness, coordination, accountability and efficiency (WHO, 2010).

High-quality health services, ensuring patient satisfaction, require proper definition of the role of patients and the use of their potential in the treatment process by engaging them in decision-making and organisational (communication) processes. The primary premise should be focus on the patient and his needs, strengthening the role of the patient, conducive to ensuring efficient communication enabling active participation of the patient in the decision-making process regarding care.

\section{Research procedure}

The starting point in the research process was the assumption that patient engagement is an important component of health services, covering a wide area of activity at the system and organisational level. In this context, it should be assumed that for the smooth functioning of the health care system, a comprehensive and coherent approach to engaging patients in decision-making about care, directed at conscious and active cooperation for the health condition, is necessary. To ensure effectiveness, a process-based approach to patient engagement management is necessary, which means targeted, comprehensive, continuous and systematic action, integrated within the entire health system and coherent with the objectives of the implemented health policy. In the article, the authors attempted to answer the following research questions:

- What is the essence of the patient engagement and his role in the health care system?

- What are the premises and conditions for managing process of patient engagement?

- What activities are key aspects of managing patient engagement in the process approach?

The article is a conceptual research study and aims to analyse the essence of patient engagement and its role in the functioning of the health care system, and to identify the premises and determinants of management aspects of involvement in a process approach. To achieve the adopted goals, the following research methods were used: literature study (a review and indepth analysis of scientific literature), document analysis (review and analysis of documents, reports and legal acts), and to explore the research problem - preliminary individual semi-structured interviews with experts (hospital managers). Based on the analysis, a conceptual model of process management of patient involvement was proposed, taking into account the main tasks and participants responsible for their implementation at the organisational level. During the analysis and evaluation of the examined process, mapping methods were used, enabling the development of graphic form of the model. For this purpose, iGRAFX software was used. The final stage of the research procedure is the formulation of conclusions and recommendations relating to the conditions for managing patient 
engagement in a holistic, integrated and consistent manner at the system level.

The essence and role of patient engagement in the health care system

One of the fundamental functions of health systems is the provision of patient-centred care. The World Health Organization defines this concept as services that are organised around the person, not the disease or the financing, which are perceived by people (being partners in their own health care) as responsive and are accepted by them, and the target population takes part in service delivery design and assessment (WHO, 2010). Patient focus is not just a guideline for health care providers on pro-quality activities. It means a way of thinking based on treating service recipients as equal partners and recognising their rights to participate in planning, developing and monitoring care to ensure that their needs are met (Health Innovation Network, 2016). This approach requires strengthening the patient's position in the system and engaging to actively participate in deciding on the treatment process. Strengthening the patient's position (patient empowerment) involves undertaking a range of activities including: facilitating patient communication with medical professionals, educating and providing access to health knowledge (promotion, raising awareness and stimulating self-criticism), enabling access to personalised medical information, supporting self-care and commitment to health management, helping them make informed decisions regarding the choice of care options (defining the problem, presenting options and consequences, value elicitation and referral to support services) and providing contacts with other patients (supporting self-education and individual responsibility, encouraging to take initiatives to help other patients) (Alpay et al, 2011).

As a consequence of this approach, the position and role of patients in the system changes radically. They become not so much recipients of health services but above all conscious participants in the treatment process. To make this possible, there must be a number of changes in the system that will enable the patient to be involved in making decisions about his own health and medical care. The concept of patient engagement means the "process of building the capacity of patients, families, carers, as well as health care providers, to facilitate and support the active involvement of patients in their own care, in order to enhance safety, quality and people-centredness of health care service delivery" (WHO 2016, p. 3). In general, it is "a broad concept that combines a patient's knowledge, skills, ability and willingness to manage his or her own health and care" (Strudwick et al, 2019, p. 4).

The broad context of this concept refers to the significant scope of impact. Engagement applies to the patients themselves, as well as their families and representatives, health professionals, operating under active partnership at various levels throughout and also to the entire health care system (direct care, organisational design and governance) to improve health and health care (Carman et al, 2013). Such multilateral cooperation is necessary because engagement is a complex concept; it can involve various aspects of care such as participation in the decision-making process, self-treatment, self-monitoring, patient education, goal setting or participation in the treatment process (Longtin et al, 2010).

This inclusive approach to patients, giving them subjectivity generates benefits for both the patient and the health system. In the individual dimension, it contributes to the improvement of treatment results, while in the institutional and systemic dimension it is a source of benefits in the areas of management, organisation (organisational culture), as well as in the economic and educational aspect. Patient engagement in their own care, as a function of improving communication between the patient and the provider and facilitating information transparency, fills the gap between patients' expectations and the services provided (Birkelien, 2017). 
This concept is recognised as a critical part of the continuously learning health care system or a necessary condition for the redesign of health care systems, the implementation of which are supported by aspects such as: a) benefits of work related to patient- and family-centred care and joint decision-making reflecting and accelerating the changing roles of patients and families in health care (patients become more active, informed and influential), b) effects in the form of better health outcomes (quality and safety of patients), as well as c) control of health care costs (Carman et al, 2013).

It is pointed out that the engagement of patients, their families or communities, is the foundation of effective management practices, and approaches to increase community members' perspectives are widely promoted in management education and professional standards (Linnander et al, 2017). In addition, it is one of the strategies that allow achieving a triple goal of improving health outcomes, better patient care and lower costs (James, 2013). Taking into account the multidimensional nature of the patient engagement, it is considered as a "meaningful and active collaboration in governance, priority setting, conducting research and knowledge translation" (SPOR, 2014, p. 5).

In conclusion, it should be emphasised that patient engagement, being the immanent feature of health services characterised by high quality, focus on the patient and effectiveness, can play an important role in improving the health system and developing health services. However, in order for the benefits of this approach to be felt by the patient, as well as participants and stakeholders of the health system, a systematic management approach is necessary, focused and collaborative, taking into account the specificity of processes involved in engaging patients.

\section{Premises and conditions of process management of patient engagement}

Restoring or maintaining the health of patients, as the goal of health systems, requires state efforts to define a framework for action and provide resources according to the policies of health, which is part of social policy. In this sense, economic efficiency and clinical effectiveness of the health system are important, but at the same time the attitudes of people using the services of this system are particularly significant (Nojszewska, 2011). Designed healthcare management systems should therefore take into account the special role of patients, their changing needs and the impact of their attitudes and behaviours on the effectiveness of medical processes.

In a systemic approach, health care management is defined as "the process of achieving health care objectives through human, financial, and technical resources includes strategic and operational management activities such as supply chain management, human resources management, performance management and improvement, financial management, and governance, without which resources cannot be effectively deployed to maximise health outcomes" (Linnander et al, 2017, p. 555). The goal of care management is to assist patients and improve their functional health status, enhance care coordination, reduce costs, eliminate duplication of services, and reduce the need for medical services (Bodenheimer and Berry-Millett 2009).

Management of patient management includes a number of processes necessary for building capacity of patients to a conscious and active participation in decision-making related to health care. The complexity and multidimensionality of these processes is related to three critical aspects of patient engagement such as: engagement activities range along a continuum (from consultation to partnership and shared leadership), engagement occurs at different levels and many factors affect patients' willingness and ability to engage (Carman et al, 2013). Patient engagement is a "process-like and multi- dimensional experience, resulting from the conjoint cognitive (think), emotional (feel), and conative (act) enactment of individuals toward their health management" (Graffigna et al, 2014, p. 1). 
Taking into account the safety aspect of health care, the World Health Organization identifies five main categories of factors affecting patient engagement, relating to: patient (demographic characteristics, health literacy), health conditions (disease severity), health care professionals (knowledge and attitudes), tasks (degree difficulties, competence challenges) and the health care setting (e.g. primary or secondary care) (WHO, 2016). Among the factors related to medical professionals that negatively affect the patient's engagement are: willingness to maintain control, time needed to train the patient and respond to his opinion, type of disease, personal beliefs, specialisation, ethnic origin and insufficient training in the concept of increasing patient engagement (Longtin et al, 2010). Therefore, at the institutional level, it becomes necessary to support workers, improve their communication skills, establishing rules, standards strengthen the organisational culture oriented on the active cooperation of the patient, his family and surroundings.

The ability and willingness of patients to engage in health matters is also significantly influenced by the cultural context of the country or community, including social norms, legal regulations, beliefs and social determinants of health (Edgman-Levitan et al, 2013). Factors with a broader impact that affect the participation of patients and their families and caregivers in health care decisionmaking processes include: development of patient-centred health care systems to ensure the improvement of the quality of treatment for all patients, the possibility of individual therapeutic treatment for each patient, better understanding of diseases and treatment effects, promoting scientific research and communicating results to the public, participation of caregivers in representing the charges and increasing awareness about health and diseases in the whole society (ELF, 2020).

The multiplicity and complexity of tasks related to patient engagement justifies a process-based approach to management, manifested in the purposefulness, coherence and wide range of activities, integrated into the entire health care management system. Process management is the "comprehensive, continuous and systematic application of appropriate concepts, methods and tools for influencing the processes taking place in the organisation, aiming at achieving the organisation's goals and the best satisfaction of the needs of its external and internal clients" (Nowosielski 2009, p. 75). For the process approach in management, the systematic treatment of the organisation, which is a set of interrelated and interpenetrating processes, is characteristic (Hammer and Champy, 1996). In process organisation, the client is in the centre of attention, and therefore the key to management are those activities that allow quick and effective identification and satisfaction, as well as creating the needs and expectations of clients (Grajewski, 2016).

By adopting this approach to managing patient engagement, it is possible to achieve the goals of the health system. This involves enabling the necessary changes such as (A Leadership, 2013):

- at the individual level - changes aimed at encouraging and supporting patients and their families to be active participants in care and decision making;

- at the level of health care teams changes ensuring preparation and support of teams for cooperation with patients, families and other members of the care team;

- at the organisational level - changes aimed at assigning accountability, encouraging partnerships and incorporating the patient and family perspective in all aspects of provider planning, implementation and evaluation of programs and services.

In this context, process management of patient engagement is a comprehensive impact on processes implemented at the system level (regulatory, supervisory, educational, research and development) and the organisational level (service, management, communication, training and education). 


\section{Key aspects of process management of patient engagement}

The adoption of process orientation for managing patient engagement is a multistage activity involving a number of participants and stakeholders of the health system, including patients, their families, communities, social organisations, government and public institutions performing tasks under health policy, health service providers, research institutions (universities) and the media. Management of engagement as a multidimensional concept requires consistency of goals and functioning at the system, institutional and direct patient relationships. The concept of process management implementation includes the following stages (Grajewski, 2016):

1. determination of the organisation's purpose,

2. identification of processes in the area of mega processes distinguished according to the client (recipient) criterion,

3. designing reference processes creating process maps,

4. development of process measures and internal market relations principles,

5. development of an IT system to support the operation of the organisation.

The goal of patient engagement management is to support the functioning of the health system, rationalise the number and effectiveness of health services, and increase the role of prevention and health promotion to reduce the need for inpatient, highly specialised treatment, which consequently improves the performance of service providers and patient relationships. Therefore, patient focused quality interventions are needed to improve health literacy, clinical decisionmaking and patient safety (Coulter and Ellins, 2007). They form the basis for identifying the main processes focused on creating value for the patient. The main areas of impact of patient engagement management are: knowledge, skills, ability and willingness of patients to manage health and care for themselves and family members; a culture of health care organisation that prioritizes and supports patient involvement and active cooperation between patients and health care providers to design, manage and achieve positive health outcomes (5 Elements 2014). Patient engagement relates to six key options for building relationships between individuals, families, communities, health care professionals and decision makers, which include: education (stimulating changes in behaviour at various levels of engagement, learning new partner communication skills), research (a key factor shaping health policy, professional practice and patient results), community health, direct care, organisational design and governance, and public policy (Edgman-Levitan et al, 2013).

After identifying the processes, it is necessary to develop their course (preparation of maps). For this purpose, graphic methods are used, including: activity flow cards, Clark charts, BernateneGrün charts, Gantt charts, schedules, classification schemes, Sankey charts, Ishikawa diagram and relationship diagram (Starbyła, 2012). Due to the complexity, variability and dynamic nature of health care processes, process mapping is not limited to the visual presentation of the analysed process, but should include social aspects such as interaction between participants (Antonacci et al, 2018).

To assess the effectiveness of implemented processes, a measurement system should be developed, under which reporting structures using multiple communication channels will be created, and the value of engagement initiatives will be evaluated, using measures related to a given process (e.g.: level of patient and/or family member representation or patient, family and project team satisfaction) and its results (e.g.: overall satisfaction with engagement activities, clinical/patient outcome improvements, number of engagement goals or objectives achieved or level of knowledge or information obtained) (Strudwick et al, 2019). 
To ensure the efficient implementation of processes related to patient engagement, an IT management support system is required. e-Health plays a special role in multilateral communication processes. The European Commission defines eHealth as "the use of ICT in health products, services and processes combined with organisational change in health care systems and new skills, in order to improve health of citizens, efficiency and productivity in health care delivery, and the economic and social value of health. eHealth covers the interaction between patients and health-service providers, institution-to-institution transmission of data, or peer-to-peer communication between patients and/or health professionals" (European Commission, 2012, p. 3). Information and communication tools and techniques form the basis for the efficient conduct of the main processes such as: education, communication and training.

\section{Modelling of Patient Engagement Management Process}

Modelling activities are helpful in process design. Process models used in process management have an analytical function and support the rationality of decision making. The purpose of modelling management systems is, in particular, to recommend a specific decision, to forecast system behaviour, and to train and improve staff in decision making (Grudzewski and Wilimowska, 2017). The components of the process model are design decisions regarding the identification of actors involved in operations, operational activities, performers of individual activities, inputs and outputs of activities, determination of the sequence of activities (dependence of activities, the possibility of parallel implementation) (Pietroń, 2011).

As a result of the analysis of the nature and conditions of process management of patient engagement, a conceptual model was developed, taking into account the identified specific features, determinants and requirements of this concept. The process management model includes the main actors of the process involved in processes at the institutional level - health care providers (decision makers and performers: management, medical personnel in direct relations with the patient (medical professionals, care coordinators), and support personnel: IT specialists, administration, etc.). This model presents the sequence of the main tasks and activities of processes implemented in the area of patient engagement management in two phases: preparation (Figure 1) and executive (Figure 2). 


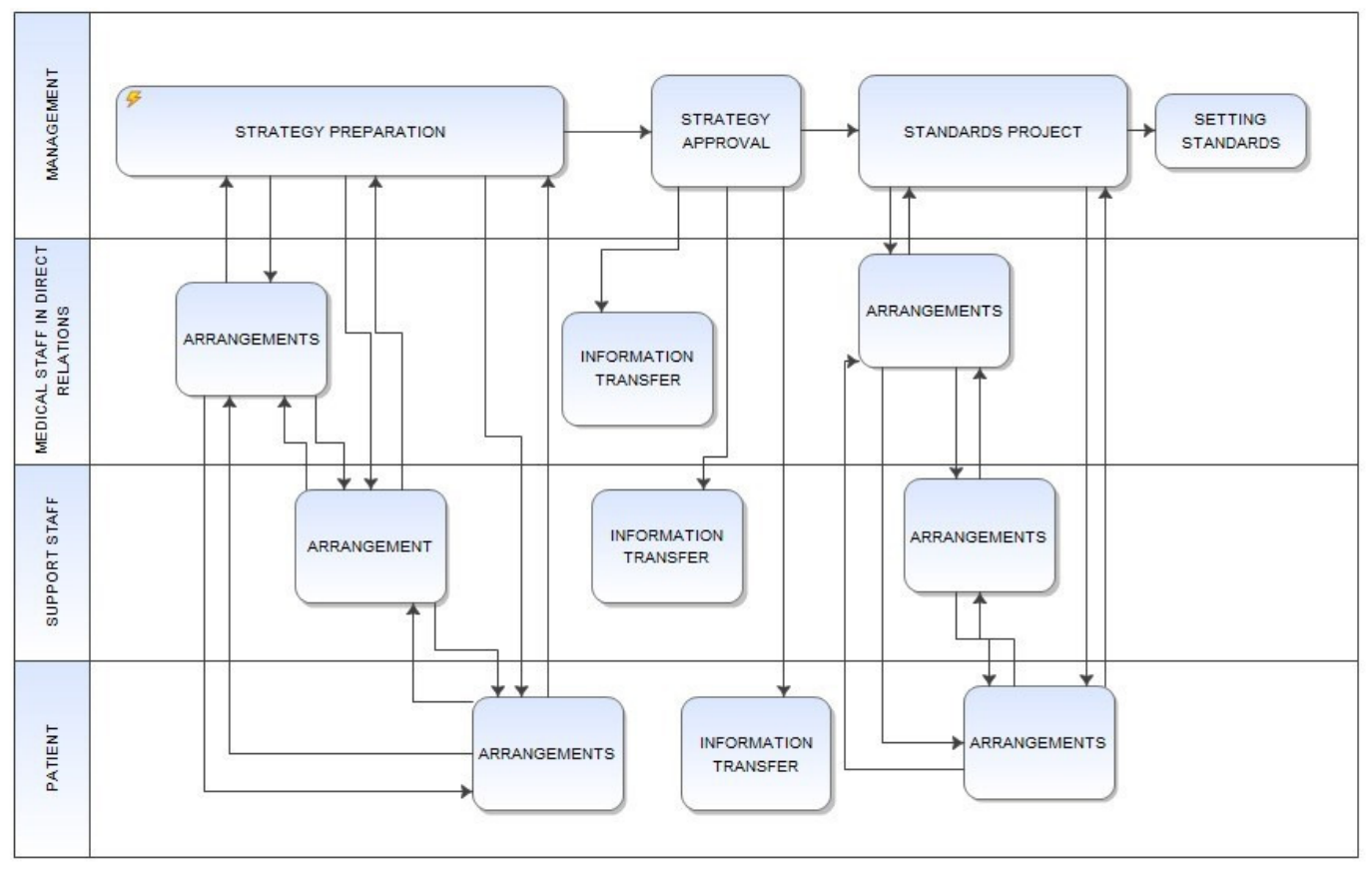

Fig. 1: The conceptual model of process management of patient engagement preparatory phase

Source: Authors analysis

Input data were determined as the need to implement process management of patient engagement in the organisation. Input data were determined as the need to implement process management of patient involvement in the organisation. The expected result (output) is to achieve patient engagement - readiness and participation in decision-making about care. To present the course of activities, a flowchart diagram was developed, using the iGRAFX program. The following stages have been distinguished in the model:

- preparation of patient engagement strategy, arrangements with employees and patients (representatives, community, patient organizations, etc.),

- strategy approval and transfer of information to all participants,

- designing standards, norms and procedures necessary to implement the strategy,
- division of tasks and activities, creation of interdisciplinary teams, appointment of leaders, care coordinators,

- preparation of an IT system to ensure communication and information and knowledge transfer,

- staff training, creating an organizational culture that supports engagement,

- preparation of programs, tools and channels of communication with the patient, education, providing ongoing personalised consultations, advice,

- strategy implementation at all levels,

- measurement and evaluation of the effects, the study values, benefits, satisfaction of patients and staff, identification of irregularities,

- implementation of adjustments and streamlining the process. 


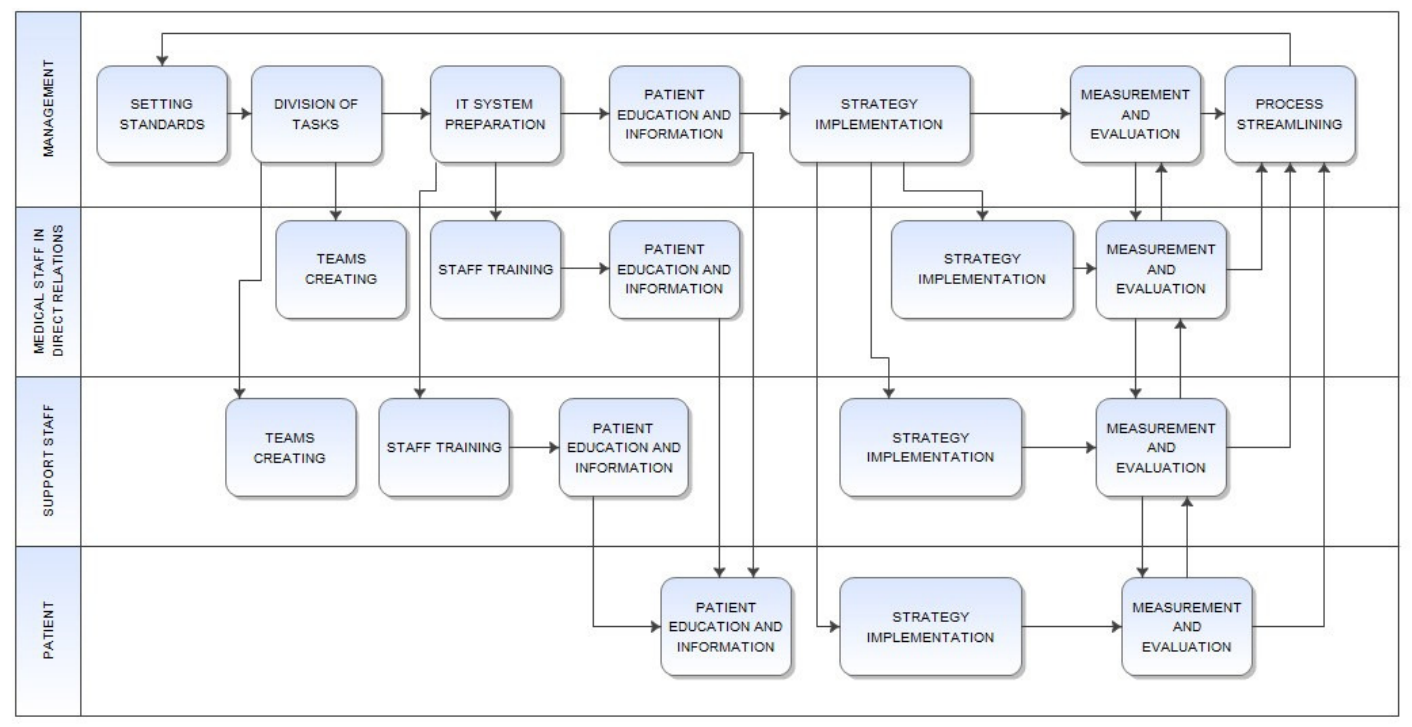

Fig. 2: The conceptual model of process management of patient

Source: Authors analysis engagement - executive phase

The proposed model is simplified and requires detailed modelling of sub processes associated with the identified main processes, but it can, however, be a starting point for designing a patient engagement management system. It should be emphasised that the usefulness of modelling, and consequently the correctness of analysing, designing and implementing the proposed systems depends on a number of factors, including the modelling methodology itself. One of the critical factors is the acceptance by all participants of the process, its goals, implementation method and measurement methods. Therefore, the participation of employee representatives, patients, their families, the community and decisionmakers are necessary in modelling the process of patient engagement management.

\section{Conclusions}

Patient engagement is an interesting concept in the scientific and practical dimension, which can contribute to increasing the quality, safety, focus on people and the effectiveness of health care services provided. However, the implementation of this concept is a challenge for health systems due to its complex, multi-entity nature. To ensure the integrity of the principles and practices necessary to implement the concept of patient engagement in all levels (the health system, health care providers, and medical professionals), a process-based approach to management is necessary. Considering the issue of patient engagement in the context of processes allows you to focus on creating and delivering value to patients and the health system. A prerequisite for engagement is patient orientation throughout the process at all levels of interaction. The organisational efficiency of service providers requires support from decision-makers in the area of legal regulations regarding norms, standards, information systems, data security, acidification and training requirements, community-wide health education campaigns, health-promoting behaviours and the possibility of influencing health care. Another important factor is the understanding and acceptance of the patient's role in the health system and a strong awareness of employees regarding their responsibility in terms of building value. In the organisation it is necessary to build strong relationships that facilitate the integration of solutions, transfer of knowledge and information, a flexible approach to the structure, creation of 
interdisciplinary teams and continuous improvement of processes taking into account the changing needs of patients and external conditions. Patient engagement is a complicated and lengthy process, but due to the specificity of health and its social and economic health value, the role of this concept will increase.

The article is valuable to identify, systematise and emphasise the role of the process approach to managing patient engagement and is the starting point for further, more detailed research.

\section{Acknowledgement}

The article was financed from the funds granted to the Faculty of Management of the General Tadeusz Kosciuszko Military University of Land Forces in Wroclaw as part of a research project financed by a subsidy granted by the Minister of National Defence of the Republic of Poland.

\section{References}

- 5 Elements of a Successful Patient Engagement Strategy, Whitepaper (2014). [Online]. athenahealth, Inc. Published. [Retrieved: September 11, 2019],

https://www.athenahealth.com/whit e-papers/patient-engagementstrategy,

- $\quad$ A Leadership Resource for Patient and Family Engagement Strategies (2013) [Online]. Health Research \& Educational Trust, Chicago. [Retrieved: September 11, 2019], www.hpoe.org/Patient-familyengagement.

- Alpay, L., van der Boog, P. and Dumaij, A. (2011) 'An empowerment-based approach to developing innovative ehealth tools for self-management,' Health Informatics Journal, 17(4), 247-255, [Online]. [Retrieved: February 8, 2020], DOI: $10.1177 / 1460458211420089$.

- $\quad$ Antonacci, G., Reed, J.E., Lennox, L. and Barlow J. (2018) 'The use of process mapping in healthcare quality improvement projects,' Health Services Management Research 0(0) 1-11, [Online]. [Retrieved: February 8, 2020], DOI: $10.1177 / 0951484818770411$.

- Birkelien, N.L. (2017) 'A Strategic Framework for Improving the Patient Experience in Hospitals. Foundation of the American College of Healthcare Executives,' Journal Healthcare Management Jul/Aug; 62(4), 250-259, [Online]. [Retrieved: January 5, 2020], doi: 10.1097/JHM-D-17-00071.

- Bodenheimer, T. and Berry-Millett, R. (2009) 'Care management of patients with complex health care needs,' Research Synthesis Report No. 19, The Robert Wood Johnson Foundation.

- $\quad$ Carman, K.L., Dardess, P., Maurer, M., Sofaer, S., Adams, K., Bechtel, C. and Sweeney J. (2013) 'Patient and family engagement: a framework for understanding the elements and developing interventions and policies,' Health Affairs 32(2), 223-31, [Online]. [Retrieved: February 8, 2020], doi: 10.1377/hlthaff.2012.1133.

- Coulter, A. and Ellins J. (2007) 'Effectiveness of strategies for informing, educating, and involving patients,' BMJ, Volume 335, 24-27.

- Edgman-Levitan, S., Brady, C. and Howitt, P. (2013), Partnering with Patients, Families, and Communities for Health: A Global Imperative. Report of the Patient and Family Engagement Working Group. WISH Patient and Family Engagement Report.

- ELF, European Lung Foundation, Patient and society involvement (in Polish), [Online]. [Retrieved: January 10 , 2020], https://www.europeanlung.org/asset s/files/pl/publications/patient_input_ polish.pdf. 
- European Commission (2012), eHealth Action Plan 2012-2020 Innovative healthcare for the 21st century, Communication from the Commission to The European Parliament, The Council, The European Economic and Social Committee and the Committee of the Regions, Brussels, 6.12.2012.

- Graffigna, G., Barello, S., Libreri, C. and Bosio, C.A. (2014) 'How to engage type-2 diabetic patients in their own health management: implications for clinical practice,' BMC Public Health 14-648, doi:10.1186/1471-2458- 14648.

- Grajewski, P. (2016) Process organisation (in Polish), Polskie Wydawnictwo Ekonomiczne, Warszawa.

- $\quad$ Grudzewski, WM and Wilimowska, Z. (2017) Od teorii do praktyki zarządzania. Czy zarządzanie jest nauką czy sztuką?, Organizacja $i$ Kierowanie 1 (175), 11-50.

- Hammer, M. and Champy, J. (1996), Reengineering in Corporation (in Polish), Neumann Management Institute, Warszawa.

- Health Innovation Network South London (2016), What is personcentred care and why is it important?, [Online]. [Retrieved: February 8, 2020],

https://healthinnovationnetwork.co $\mathrm{m} /$ system/ckeditor_assets/attachme nts/41/what_is_person-

centred_care_and_why_is_it_importan t.pdf.

- James, J. (2013) 'Health Policy Brief: Patient Engagement,' Health Affairs, February 14, 1-6. DOI: 10.1377/hpb20130214.898775

- Kovacic, L. and Jaksic, Z. (2013) Health care: levels and limits, [in:] Forum for Public Health in South
Eastern Europe. A Handbook for Teachers, Researchers and Health Professionals (2nd edition), Vol. I, Health: Systems - Lifestyle - Policies, Editors: G. Burazeri, L. Zaletel Kragelj, Jacobs Publishing Company, Lage, Germany.

- Kruk, ME. and Freedman, LP. (2008), 'Assessing health system performance in developing countries: A review of the literature,' Health Policy 85, 263276 ,

doi:10.1016/j.healthpol.2007.09.003.

- Linnander, EL., Mantopoulos, JM., Allen, N., Nembhard, IM. and Bradley, EH. (2017) 'Professionalizing healthcare management: a descriptive case study,' International Journal Health Policy Management 6 (10), 555-560, doi:10.15171/ijhpm.2017.40.

- Longtin, Y., Sax, H., Leape, LL., Sheridan, SE., Donaldson, L. and Pittet, D. (2010) 'Zaangażowanie pacjenta: stan wiedzy i wpływ na bezpieczeństwo opieki,' Medycyna po dyplomie, 19 (6), 117-133.

- Nojszewska, E. (2011) Health care system in Poland (in Polish), Wolters Kluwer Polska Sp. z o.o., Warszawa.

- $\quad$ Nowosielski, S. (ed.) (2009) Process approach in organisations (in Polish), Uniwersytet Ekonomiczny w Krakowie, Kraków.

- Pietroń, R. (2011) Process Management, Business Information Systems, Wroclaw University of Technology, Wroclaw.

- SPOR (2014), Strategy for PatientOriented Research. Patient Engagement Framework, The Canadian Institutes of Health Research, the SPOR Patient Engagement Consultation Workshop (on January 9, 2014).

- Stabryła, A. (2012) 'Ogólna koncepcja analizy i projektowania systemów 
zarządzania procesowego,' Zeszyty Naukowe Małopolskiej Wyższej Szkoły Ekonomicznej w Tarnowie, 21 (2), 125-142.

- $\quad$ Strudwick, G., Leung, K., McLean, D., Booth, R., Borycki, E., Rossetti, S., Kuziemsky, C., Zhang, T. and Kemp, J. (2019) Patient and family engagement in health information technology initiatives: Findings of a literature review, focus groups and symposium. Toronto: Centre for Addiction and Mental Health.

- WHO, World Health Organization (2000), Health systems: improving performance, The World health report 2000, [Online]. [Retrieved: February
3 , 2020],https://www.who.int/whr/200 $0 /$ en/.

- WHO, World Health Organization (2010), Monitoring the building blocks of health systems: a handbook of indicators and their measurement strategies, section 1, Health service delivery. [Online]. [Retrieved: February 3, 2020], http://www.who.int/healthinfo/syste ms/WHO_MBHSS_2010_section1_web .pdf.

- WHO, World Health Organization (2016), Patient Engagement: Technical Series on Safer Primary Care, Geneva. 